\title{
Design and application of the network distance teaching system
}

\author{
Pengbin, Hongxueqiong, Pengjingxin \\ NanChang University GongQing College \\ Gongqing, 332020, China \\ 35460510@qq.com
}

\begin{abstract}
Computer network generate a spatial and temporal separation in teaching activities, the new feature of the online teaching also make it possible to improve students' learning efficiency by using of the theory of interactive activities and appropriate teaching strategies. This paper mainly discuss the problems exist in distance teaching system based on the Internet, and design an prototype model for distance teaching which could achieve a unified and efficient management of curriculums, teachers, students and teaching resource according to the specific requirement of school.
\end{abstract}

Keywords-Internet, Distance teaching, System design, Database

\section{INTRODUCTION}

The final goal of teaching and learning is to achieve the desired learning target which can be realized only through the study of students. As we know, learning is a internal psychological cognitive processes of students. Only outside learning content and information enter the learning process, can the final learning outcomes formatted[1 3]. Therefore, we believe that centering on the learning process does help to combine teaching and learning, play the leading role of teachers and reflect the dominant position of students in learning activities. Distance teaching system, centers around learning process, is described as follow: Learning process points to learning goals[4 6]. To ensure the learning process achieve the learning goals successfully, a balance between the control from the teaching institutions and the learners, that is to maintain an appropriate ratio. When changes, which is fed back through a variety of communication to teaching institutions, appear in learners' intervention of the learning process, the conduct of the learning process would be affected. Once teaching staff receive the feedback, they would adjust the proportion of teaching and learning strategies in various media in order to adjust the entire proportion of the strategies. Besides, teaching staff could affect the learning motivation and cognition strategies of learners. Through these two ways, teaching staff adjust themselves and the intervention of learners in the learning process, so that the whole system is in a dynamic equilibrium. In the whole process, learning intervention is obtained through adjustment of the proportion of strategies centered around teaching and strategies centered around learning to achieve control of the intervention strength of teaching in learning process. In the system, we package strategies centered around learning and teaching in the medias through which transmit the teaching strategies to learners to intervene in the learning process[7].

\section{PROBLEMS DESCRIPTION}

In network distance teaching system, CAI courseware, replaces textbook and blackboard, is the direct source of knowledge of the students. So the quality of CAI undoubtedly plays an important role. Currently, CAI courseware are made by teachers and sent to network management center, then published on the internet. The whole process is confusing owing to lack of uniform standards. In addition, to lots of teachers, CAI courseware design is not a easy work. Different teachers make CAI courseware with different quality. Several teachers even just scan and paste the textbook which greatly lower the quality of courseware. Furthermore, network security has become a specialized discipline containing a wide range of content. Here we emphasize digital signature technology and data protection technology which are particularly prominent objects in network security for distance teaching system. To begin with, students enter the distance teaching system by username and password. Most recent distance teaching system only has one username, always the name of the teaching system or the university. Passwords are unified arrangement by school, general are students' number or a combination of students' number and class name. Such kinds of username and password can be stolen easily, resulting in unnecessary losses and troubles to the students, and disrupting the normal order of teaching. In addition, the courseware, handouts, test questions and answers are accumulated in teachers' years of teaching experience what should be included in the scope of protection of intellectual property rights. But many distance teaching system publish the information web without any data protection and free to copy, reprint and print. It is a serious violation of the intellectual property rights, and dampen the enthusiasm of teachers.

\section{Ystem Design Based On .NET Technology}

\section{A. System structure}

Network distance teaching platform based on B/S structure. WEB server stores the entire Web application programs of the network distance teaching platform, while database server stores the data information associated with online teaching and system management, such as user account, the Q \& A Forum information, the student work 
information, examination pool and record, teaching and research searching information. Teachers, students and administrators given different access rights[1] access the application programs server and database of the system through browser based on Internet. The network topology of the system is shown in Figure 1

Network distance learning platform has a high specialization, large amount of data processing, complex functions, variability and high requirements of security and interaction. Considering all above, the system adopts three layers structure, including presentation layer, business logic layer and data layer. The presentation layer is the interactive interface between users and system, composed by Web forms, users' interface element and so on. The main purpose of presentation layer is to obtain and display data from business logic layer firstly, then interact with users, finally send related data back to business layer. According to the customer request, business logic layer realize role management, competences distribution, authentication identity and application, mission decision-making, transaction processing and process control in the process of teaching management. At the same time, business logic layer manages all the database access rules, determine the way of service management to ensure security. Data layer contains storage data and components or services interacted. Database mainly uses the relational database, to ensure the integrity, consistency of system data through rule definition, constraint, transaction, concurrency control mechanism and so on. The architecture of the system is shown in figure 2 .

In this mode, make full use of the separation of .NET technology front page and the background code, then use .aspx page to display content and DLL generated by code compiling for data processing to generate dynamic content. Manage business layer and data layer through background DLL programs, then separate the large amounts of data \& business logic and page display, and there is no need for aspx page layer to process any business logic. In the purposed system, large amounts of pages need to be displayed through asp.net data-bound controls. Then the system call back users' requests through asp.net programs and refresh the page quickly. This mode can separate the content generation from content display more simply and make the actual software development more efficient \& business logic clearer than the previous web authoring \& Application mixing performance.

In Web application systems, data presentation is a main factor restricts system across platform and update. The versatility and compatibility of the system will be greatly enhanced if data information can be freely transferred and exchanged between information products with different nature on different platforms. XML (eXtensible Markup Language) is a language designed for information selfdescription, which focus on how to describe the organization and structure of data content used for net communication and processing. It separates user interfaces and structured data, allows seamless integration of data from different sources and multiple data processing. It is flexible, scalable, well structured and constraint, furthermore, a natural way to represent and exchange heterogeneous data[3].
In traditional Web application program model, users need to submit the form to achieve page exchange. This click and wait mode forces users to interrupt work process and wait for page reload which badly affect the visual effects and interaction of Web pages. AJAX (Asynchronous JavaScript And XML) send and receive asynchronous request/response of the server through communication technology (general SOAP and XML), then process them with display technology (JavaScript, DOM, HTML and CSS).[4] It allows flexible partitioning of application logic and the state between the client and server management, which not only eliminate the "Click - wait" dependent, but also provide a better server scalability. ASP.NET provides a number of built-in controls through which rich, interactive personalization AJAX-style applications can be compiled and it realize the seamless integration of browser UI and other applications convenient for development and debugging.

\section{B. System function module}

The network distance teaching platform is composed by three modules: teaching module, students module and administrator module. The three modules, relatively independent and associated with each other, form a perfect function, flexible operation network distance teaching platform. The function of teacher module are corresponding to the function of the student module, and the administrator module is used to manage personnel, courses, classes and courses to open of the network distance teaching platform. The system function modules are shown as figure 3:

\section{Web Service}

World Wide Web is the mainstream application in Internet. IIS (Internet Information Server) from Microsoft is a Web server with complete Web characteristics. Generally the Web browsers use HTTP (Hyper Text Transfer Protocol) protocols to communicate with Web servers. HTTP supports the transmission of hyper texts and other files (including video and audio) in WWW. The details are as follows. First, the client Web browser opens a Web server connection, sends a Web page request which is normally the GET or POST request in HTTP. GET request can search static pages, images or other Web objects such as audio or video. Then the browser waits for the responses. Second, the request can be sent to responding Web server to process via Internet. The targeted server applies Uniform Recourse Locator (URL) to identify the domain. Web servers receive the requests, generate a response to client and close the connection, which searches for the files with the same directory in the URL and response to Web browser. In order to help the browsers get familiar with the types of transmitted files, the server must transmit the header information of Content-Type. Finally, Web browser receives the files and displays the images according to the HTML (Hyper Text Makeup Language) codes.

\section{Database support}

Modern distance education cannot be separated from the database management technology, which includes storage \& 
query of many educational data, and information associated with curriculum, student learning, teachers counseling, so the application of distance database system is very important. Currently, Web server access database mainly through CGI, API for Web server, ADO/ASP and so on. CGI (Common Gateway Interface) is a platform-independent communications standardization used to transfer information among Web browser, Web server and CGI application programs. CGI programs can be compiled in various programming languages and scripting languages, such as Perl, C/C++, VB. But it is low efficiency and difficult updated for CGI to access Web database. Therefore, in order to extend the function of server, almost all commercial Web server provide special used API (SAPI). For example, NSAPI for Netscape, ISAPI for Microsoft IIS. Generally speaking, SAPI are provided in pattern of DLL. Application using such SAPI is compiled into a DLL, thus becomes part of the Web server application called directly by the Web server. The speed and performance has been greatly improved, but the main drawback is the compatibility. Another technique is to use ADO/ASP. ADO (ActiveXData Object) is database access component in ActiveX component using OLE DB for its foundation and language independent components technology to provide a high level of packaging for OLE DB, thereby shield the basement details. ASP (Active Server Page), a component in Microsoft Web server IIS, provides a server-side scripting environment. Efficient Web database access can be realized through applying ADO component technology in ASP web development project.

\section{CONCLUSION}

Network distance education, has characteristic of rich educational resources and collaborative learning, are gaining the generally recognized of the education community. Comprehensive evaluation can determine whether students learning course materials required, both of online quizzes and class exams can achieve different purposes. The online quizzes materials are sent to students by teachers, which should be finished by students in proper time.

Teachers correct test papers timely, and send the results back to students. Many distance teaching system allows learners to answer the questions online. When completed, the answer could be submit by click the accomplish button. Teachers could comment on each answer through this kind of test method. Distance web-based network teaching mode will be recognized by people and become a common way of distance education. Combined with cable TV, distance webbased network teaching mode will also become backbone force in network distance education.

\section{REFERENCES}

[1] Carreras X , Marquez L. Boosting Trees for Anti-Spam Email Filtering[C]. In : Proceedings of Euro Conference Recent Advances in NLP (RANLP-2001). 2001: 58-64.

[2] Cohen W. Fast Effective Rule Induction[C]. In Machine Learing: Proceedings of the Twelfth International Conference, Lake Taho , California , Mongan Kanfmann , 1995:115-123.

[3] Cohen W. Learning Rules that Classify email[C]. In Proceedings of the AAAI spring symposium of Machine Learning in Information Access , Palo Alto, California , 1996:18-25.

[4] Drucker H, Wu D, Vapnik V N."Support Vector Machines for Spam Categorization”[J]. IEEE Transactions on Neural Networks. 1999 , 20(5):1048-1054.

[5] Nicholas T. Using AdaBoost and Decision Stumps to Identify Spam E-mail [EB] . Stanford University Course Project (Spring 2002/2003) Report, cs224n/2003/fp/tyronen/report.pdf. http://nlp.stanford.Edu/courses/

[6] Androutsopoulos I, Paliouras G , Michelakis E. Learning to Filter Unsolicited Commercial E-Mail [EB] . Technical report 2004/2 , NCSR"Demokritos”, 2004.

[7] Pawlak Z. Rough set [J]. Inter Journal of Computer and Information Sciences, 1982, 11: 341-356.

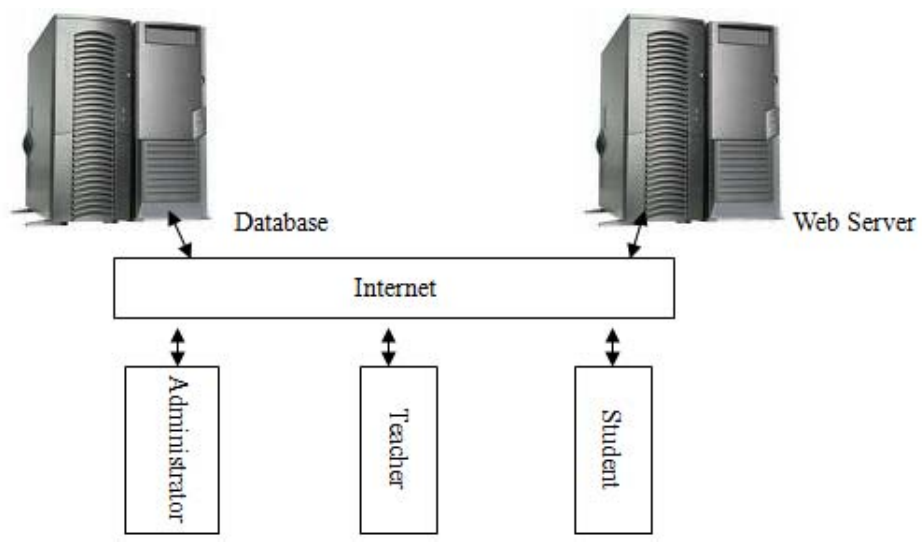

Figure 1. The network topology of the system 


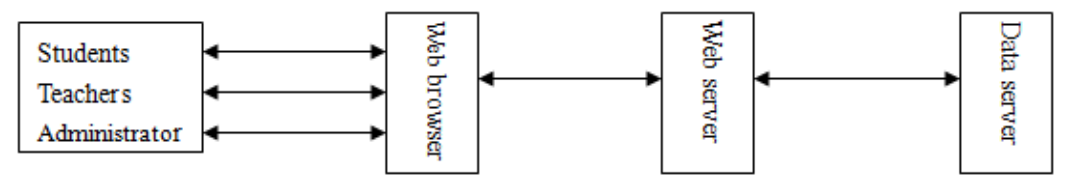

Figure 2. The architecture of the system

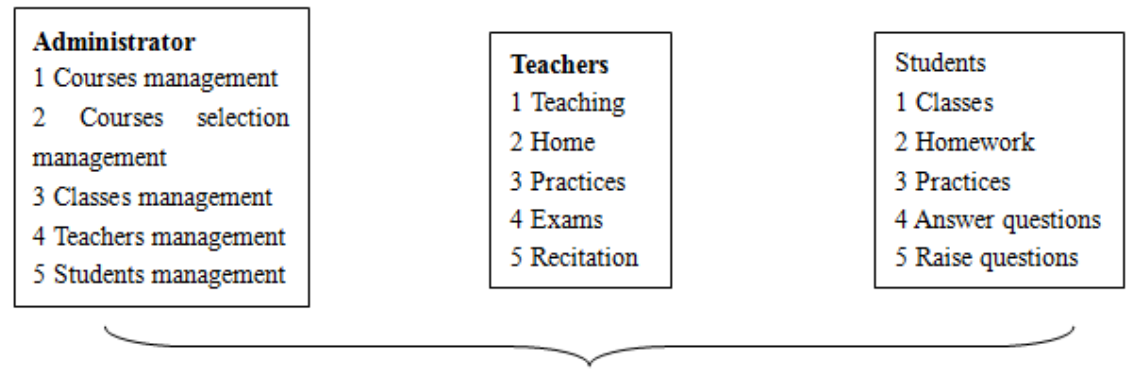

Figure 3. Network Distant teaching system functional modules 